\title{
Young's modulus, Poisson's ratio, and residual stress and strain in (111)-oriented scandium nitride thin films on silicon
}

\author{
M. A. Moram, ${ }^{\text {a) }}$ Z. H. Barber, and C. J. Humphreys \\ Department of Materials Science and Metallurgy,University of Cambridge, Pembroke Street, \\ Cambridge CB2 3QZ, United Kingdom
}

T. B. Joyce and P. R. Chalker

Department of Engineering, Materials Science and Engineering, University of Liverpool, Liverpool L69 3GH, United Kingdom

(Received 16 August 2005; accepted 25 May 2006; published online 21 July 2006)

\begin{abstract}
Epitaxial scandium nitride films (225 nm thick) were grown on silicon by molecular beam epitaxy, using ammonia as a reactive nitrogen source. The main crystallographic orientation of $\mathrm{ScN}$ with respect to $\mathrm{Si}$ is $(111)_{\mathrm{ScN}} \|(111)_{\mathrm{Si}}$ and $[1-10]_{\mathrm{ScN}} \|[0-11]_{\mathrm{Si}}$; however, some twinning is also present in the films. The films displayed a columnar morphology with rough surfaces, due to low adatom mobility during growth. The strain-free lattice parameter of $\mathrm{ScN}$ films grown under optimized conditions was found to be $4.5047 \pm 0.0005 \AA$, as determined using high-resolution X-ray diffraction (HRXRD). In-plane and out-of-plane strains were subsequently evaluated using HRXRD and were used to determine the Poisson ratio of $\mathrm{ScN}$ along the $\langle 111\rangle$ direction, which is found to be $0.188 \pm 0.005$. Wafer curvature measurements were made and combined with the strain information to determine the average Young's modulus of the films, which is found to be $270 \pm 25 \mathrm{GPa}$. Residual film stresses ranged from -1 to $1 \mathrm{GPa}$ (depending on film growth temperature and film thickness) due to competition between the tensile stress (induced by the differential thermal contraction between the $\mathrm{ScN}$ film and the $\mathrm{Si}$ substrate) and intrinsic compressive stresses generated during growth. (C) 2006 American Institute of Physics. [DOI: 10.1063/1.2217106]
\end{abstract}

\section{INTRODUCTION}

$\mathrm{ScN}$ is a semiconducting transition metal nitride possessing the rocksalt structure. Previous work has shown it to possess a direct band gap of 2.1-2.4 eV (Ref. 1) and a lattice constant of 4.500-4.505 $\AA^{1,2}$ which represents a mismatch of as low as $-0.1 \%$ to GaN. Additionally, as it has a high melting point of $2600{ }^{\circ} \mathrm{C}$ (Ref. 1) and does not decompose or undergo any reactions or phase transformations under the conditions employed for growth of other nitride semiconductors, there is considerable potential for combining $\mathrm{ScN}$ with materials such as AlN, GaN, and $\mathrm{InN}$ for device applications. Silicon is the preferred substrate for device growth as it is cheap and readily available in large wafer sizes. However, due to the effect of differential thermal contraction, thin film device structures grown on silicon substrates often display high levels of residual tensile stress, which is induced upon cooling from the growth temperature. Knowledge of the Poisson's ratio and the Young's modulus of (111)-oriented $\mathrm{ScN}$ films is desirable in order to assess the likely stress development if such films were incorporated as part of an electronic device based on a silicon substrate. Residual stresses and strains are known to affect device performance [for example, residual strains may affect the wavelength of light emitted from a GaN-based light-emitting diode (Ref. 3)] as well as influencing the ultimate attainable thickness of a thin film device heterostructure; excessive residual stress levels may result in the cracking or spalling of epilayers.

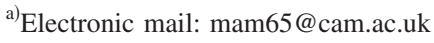

Accurate determination of residual stresses and strains is also necessary to understand stress development during growth, which can affect film microstructure and crystalline quality.

The elastic properties of $\mathrm{ScN}$ films on Si have therefore been investigated by determining macroresidual strains in a series of $\mathrm{ScN}$ films using high-resolution x-ray diffraction (HRXRD). The strain information allows the strain-free lattice parameter and the Poisson's ratio of the films to be determined. By combining strain measurements with HRXRD wafer curvature measurements, values for the Young's modulus of the films were acquired, allowing evaluation of stresses in the deposited films. Information on the morphology and roughness of the films was obtained in order to facilitate comparison with previous values of Young's modulus obtained for (100)-oriented ScN films.

\section{EXPERIMENT}

\section{A. Film growth, morphology, and roughness}

$\mathrm{ScN}$ films were deposited on (111)-oriented Si substrates using molecular beam epitaxy (with $\mathrm{NH}_{3}$ as a nitrogen source) at temperatures ranging from 600 to $1000{ }^{\circ} \mathrm{C}$. The optimum film growth conditions were determined previously and described in detail elsewhere. ${ }^{4}$ The current study employs films grown at different temperatures in order to investigate the effects of residual stress. The morphology of the films was investigated using transmission electron microscopy (TEM). Cross-sectional samples were prepared for TEM by mechanical thinning to approximately $60 \mu \mathrm{m}$, followed by dimpling to a thickness of $25 \mu \mathrm{m}$, and finally ion 


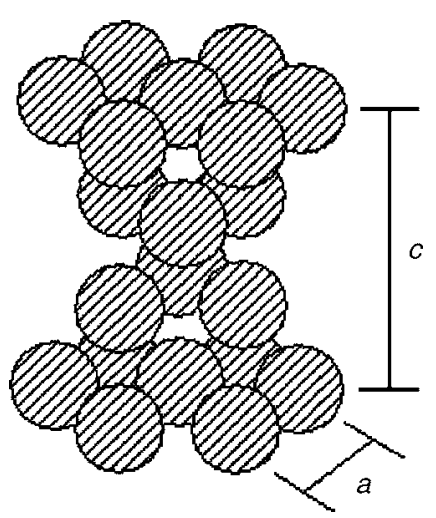

FIG. 1. Illustration of the fcc stacking sequence in a (111)-oriented ScN film, with the nonprimitive hexagonal cell parameters chosen for strain determination outlined. For simplicity, only one type of atom is shown, although the $\mathrm{NaCl}$ structure consists of two interpenetrating fcc sublattices.

milling to electron transparency. The roughness of the films was evaluated using atomic force microscopy (AFM).

\section{B. Strain measurements}

Lattice parameters were determined at $25^{\circ} \mathrm{C}$ using a Philips X'Pert Gen 6 high-resolution X-ray diffractometer in the triple axis configuration, with an asymmetric $\mathrm{Ge}$ quadruple-bounce monochromator (removing $\mathrm{Cu} K \alpha_{2}$ and $\mathrm{Cu} K \beta$ radiation) and a triple-bounce analyzer. ${ }^{5} \mathrm{Cu} K \alpha_{1}$ radiation of wavelength of $1.540598 \AA$ was used in calculating lattice parameters. The diffractometer setup was optimized for accurate lattice parameter measurements, including the use of a specimen height positioning stage that could be set to within $0.001 \mathrm{~mm}$. A resolution of $0.0001^{\circ}$ in $\theta-2 \theta$ scans and $0.01^{\circ}$ in $\varphi$ and $\psi$ scans was available, where $\varphi$ refers to rotation about an axis lying within the plane of the sample and $\psi$ refers to rotation about an axis lying perpendicular to the plane of the sample. The peaks displayed a fully Lorentzian profile and hence a Lorentzian curve was fitted to each peak to determine its position.

HRXRD measurements of strain involve accurate measurements of interplanar spacings, which are used as strain gauges. (111)-oriented cubic films undergo a rhombohedral (trigonal) distortion when in-plane biaxial stress is present (such as that resulting from differential thermal contraction between the film and the substrate upon cooling from the film growth temperature). In-plane lattice parameter measurements are awkward to determine when the conventional cubic indexing is retained, due to this unit cell distortion. In order to facilitate in-plane and out-of plane strain measurements, an alternative nonprimitive unit cell was chosen to describe the film's crystal structure, as illustrated in Fig. 1. This hexagonal unit cell was chosen to have its $c$ axis perpendicular to the film surface (the $c$ unit cell parameter being equal to the fcc stacking repeat distance) and its $a$ axis parallel to the film surface [the $a$ parameter being equal to the distance between like atoms in the close-packed (111) plane]. The $a$ and $c$ parameters are related to the conventional cubic unit cell by $a=\sin 45^{\circ} d_{100}$ and $c=3 d_{111}$. Using the nonprimitive unit cell, the $c$ parameter was determined from $d_{111}$ (cubic) measurements, which provide out-of-plane strain in- formation. These were taken from measurements of the symmetric (333) cubic reflection wherever possible, to improve accuracy. The $a$ parameter was then found from asymmetric reflections by measuring the average $d_{311}$ (cubic), which is equal to $d_{105}$ (hexagonal) and confirmed by measuring the average $d_{200}$ (cubic), which is equal to $d_{1-12}$ (hexagonal). This distance could be entered into Eq. (1) to find $a$, and hence the in-plane strain, ${ }^{5}$

$$
\frac{1}{d^{2}}=\frac{4}{3}\left(\frac{h^{2}+h k+k^{2}}{a^{2}}\right)+\frac{l^{2}}{c^{2}} \text {. }
$$

The strain-free lattice parameter is also required to obtain actual strain values. In order to minimize error, a method involving the direct use of measured lattice plane spacings (rather than residual stress measurements using the Stoney equation, which may be subject to considerable uncertainty) was employed. For an unstrained cubic structure, the ratio of $d_{111}: d_{311}$ is equal to $1:(\sqrt{ } 3 / \sqrt{ } 11)$. Strained cubic films will give $d_{111}: d_{311}$ ratios that deviate from this. The value of $d_{111}$ can be plotted versus measured ratios to find a value for the strain-free distance $d_{111}$ corresponding to the ideal $d_{111}: d_{311}$ ratio, hence allowing the strain-free lattice parameter to be obtained.

A (111)-oriented film having the rocksalt structure should possess in-plane isotropy. Therefore, although the single-crystal material is elastically anisotropic, the in-plane stresses and strains can be described using equations applying to isotropic materials. A plot of the out-of-plane strain $\left(\varepsilon_{c}\right)$ versus the in-plane strain $\left(\varepsilon_{a}\right)$ can be used to obtain the Poisson's ratio $(\nu)$ along the $\langle 111\rangle$ direction, using the following relationship, which assumes a state of in-plane equibiaxial strain. ${ }^{6}$

$$
\varepsilon_{c}=-\frac{2 \nu}{1-\nu} \varepsilon_{a}
$$

\section{Stress determination}

Stresses in thin films are often estimated from wafer curvature measurements using the Stoney equation. ${ }^{7}$ An alternative form of the Stoney equation is used in this paper, which is modified to include the effect of initial substrate curvature, the effect of nonzero film thickness, and the effect of differing Young's moduli of film and substrate, ${ }^{8}$

$$
\sigma_{f}=\frac{E_{s}{ }^{\prime} t_{s}^{2}}{6 t_{f}}\left(\frac{1}{R}-\frac{1}{R_{0}}\right)\left(1+4 E_{r}{ }^{\prime} t_{r}-t_{r}\right) .
$$

$\sigma_{f}$ is the film stress, $R_{0}$ and $R$ are the radii of curvature of the substrate pre- and postdepositions, respectively, and the ratio of film to substrate thicknesses is given by $t_{r}=t_{f} / t_{s}$. For the case of a spherically curved plate, the effective elastic moduli are given as follows: $E_{s}{ }^{\prime}=E_{s} /\left(1-\nu_{s}\right), E_{f}{ }^{\prime}=E_{f} /(1$ $-\nu_{f}$ ), and $E_{r}{ }^{\prime}=E_{f}{ }^{\prime} / E_{s}{ }^{\prime}$, where $E_{f}$ and $E_{s}$ are the Young's moduli of the film and the substrate, and $\nu_{f}$ and $\nu_{s}$ are the Poisson's ratios of the film and substrate, respectively. The value of the in-plane Young's modulus for the film is clearly required in order to evaluate stress. This is unknown; therefore, a second, independent method to determine stress is required in order to obtain the in-plane Young's modulus 


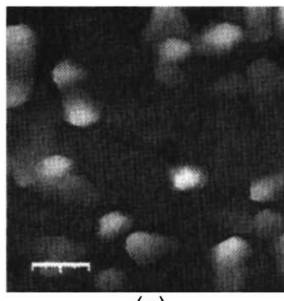

(a)

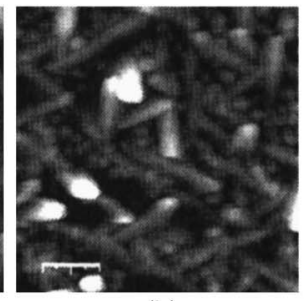

(b)

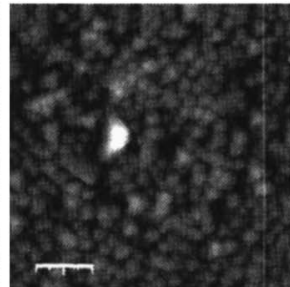

(c)

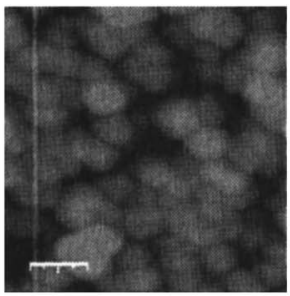

(d)

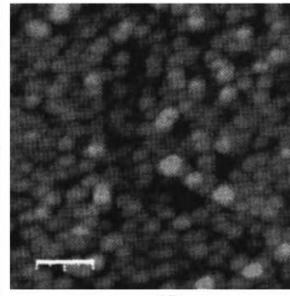

(f)

FIG. 2. AFM images of $\mathrm{ScN}$ films grown at (a) $600{ }^{\circ} \mathrm{C}$ (grayscale $=123 \mathrm{~nm}$ ), (b) $700{ }^{\circ} \mathrm{C}$ (grayscale $=91 \mathrm{~nm}$ ), (c) $800{ }^{\circ} \mathrm{C}\left(\right.$ grayscale $=86 \mathrm{~nm}$ ), $\left(\right.$ d) $900{ }^{\circ} \mathrm{C}$ (grayscale $=65 \mathrm{~nm}$ ), and $(\mathrm{e}) 1000^{\circ} \mathrm{C}$ (grayscale $=42 \mathrm{~nm}$ ). The scale bars correspond to $200 \mathrm{~nm}$.

(and hence the film stress). Assuming in-plane isotropy, the following expression is used:

$$
\sigma_{f}=-\frac{E}{2 \nu} \varepsilon_{c}
$$

where $\sigma_{f}$ is the in-plane film stress and $\varepsilon_{c}$ is the out-of-plane film strain. ${ }^{9}$ Out-of-plane strain is employed, as it can be determined with higher accuracy than in-plane strain due to the narrower and more intense nature of the $\mathrm{x}$-ray diffraction peaks of the symmetrical (111) reflection compared to those of the asymmetrical (200) or (311) reflections. This expression can be used in conjunction with the previously determined Poisson's ratio to provide a second expression relating film strain to film stress, which also includes the Young's modulus of the film. Equating the two expressions allows the Young's modulus to be obtained, followed by the stress.

The modified Stoney equation describes the stress state for the case of epitaxial films under biaxial stress with decreasing accuracy as wafer curvature increases. ${ }^{8}$ Therefore, only films under relatively low strains (and presumably, therefore, under low residual stresses) were used for the determination of the elastic constants. This also avoids the unwitting use of data from films that may be in the plastic regime. To avoid discrepancies in data arising from the difference in microstructure between films deposited at different growth temperatures, elastic constants were determined using data from a series of films of different thicknesses, grown under identical optimized conditions at $850{ }^{\circ} \mathrm{C}$. In order to improve the accuracy of stress values calculated from the modified Stoney formula, the reduced modulus of our silicon substrates was measured using analysis of the loading-unloading curves obtained during a series of 50 nanoindentation tests (MicroMaterials NanoTest indentation equipment, loads of 50-200 mN applied using a Berkovich indenter). ${ }^{10}$ Taking into account the elastic properties of the indenter tip, an accurate value of Young's modulus for the doped $\mathrm{Si}(111)$ substrates used in this study was then determined to be $125 \pm 5 \mathrm{GPa}$. The thickness of each wafer was individually measured (using optical microscopy of the wafer cross section) to within $\pm 5 \mu \mathrm{m}$. The radii of curvature of both the bare silicon substrates (prior to deposition) and of the wafers with films deposited on them were measured to within $\pm 0.05 \%$, using a HRXRD technique that directly measures the curvature of the diffracting lattice planes parallel to the substrate surface.

Shear strains cannot be directly measured using XRD and have been ignored in this treatment. If the films display in-plane elastic isotropy, no shear strains should result if a rotationally symmetric biaxial stress is applied. However, even if the stress is not perfectly rotationally symmetric, this assumption is still justified in our case, as transition metal nitrides are known to strongly resist shearing strains. ${ }^{11}$

\section{RESULTS AND DISCUSSION}

\section{A. Microstructure}

A series of (111)-oriented $\mathrm{ScN}$ films (225 $15 \mathrm{~nm}$ thick) were grown on (111)-oriented $\mathrm{Si}$ substrates at temperatures between 600 and $1000{ }^{\circ} \mathrm{C}$. Data on the $\mathrm{ScN}$ film orientation and crystallinity have been reported elsewhere, ${ }^{4}$ showing that the $\mathrm{ScN}$ films are of good crystalline quality (as evidenced by narrow rocking curves of the $\mathrm{ScN}$ (111) peak), but are partially twinned. An optimum growth temperature of $850{ }^{\circ} \mathrm{C}$ was identified previously. ${ }^{4}$ The principal orientation of the $\mathrm{ScN}$ unit cell is rotated $60^{\circ}$ with respect to that of the $\mathrm{Si}$ substrate, giving an orientation relationship of $(111)_{\mathrm{ScN}} \|(111)_{\mathrm{Si}}$ and $[1-10]_{\mathrm{ScN}} \|[0-11]_{\mathrm{Si}}$, whereas the minor twinned component displays a cube-on-cube relationship with the substrate.

The morphology of the films varies with growth temperature, with uniform growth only occurring above $800{ }^{\circ} \mathrm{C}$, as shown in Fig. 2. A similar reduction in uniformity can be induced by growing films at $800{ }^{\circ} \mathrm{C}$ but under decreased $\mathrm{NH}_{3}$ flow rates; the nonuniform morphology is therefore attributed to Sc-rich growth conditions at low temperatures due to the limited $\mathrm{NH}_{3}$ cracking efficiency. This suggests that even the optimized films are likely to have been grown under stoichiometric or slightly Sc-rich conditions, rather than under N-rich conditions.

TEM micrographs were obtained for a $430 \mathrm{~nm}$ thick film grown at $900{ }^{\circ} \mathrm{C}$ (Fig. 3) which was previously determined, using XRD pole figures, to have just $2.65 \pm 0.05$ vol \% of twins (these are localized at the interface between the film and the substrate). ${ }^{4}$ A selected area diffraction pattern (Fig. 3, inset) taken over the whole film cross section confirms its single-crystal orientation, with no diffraction spots attributable to other orientations of $\mathrm{ScN}$. The film shows no evidence of porosity, although a columnar microstructure is observed, with the columns delineated by sharp facets. Others have also obtained rough (111)-oriented $\mathrm{ScN}$ films grown on both sapphire and $\mathrm{MgO} .{ }^{1,12}$ However, Gall et al. have reported the realization of smooth (100)-oriented films on $\mathrm{MgO}$ (grown at the similar temperatures to our films) that do not display such a microstructure. ${ }^{13}$ Evidence has previously 


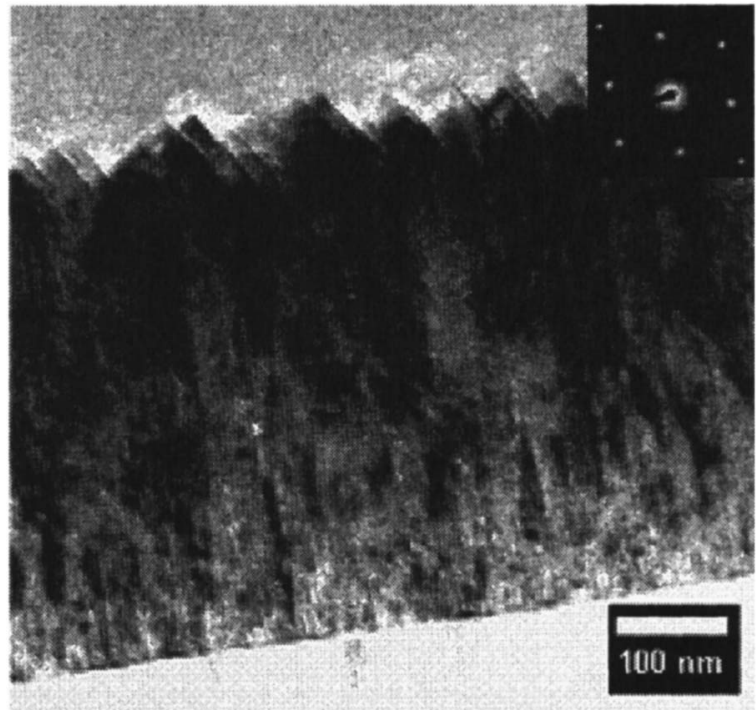

FIG. 3. Cross-sectional TEM image of a $430 \mathrm{~nm}$ thick $\mathrm{ScN}$ layer grown at $900{ }^{\circ} \mathrm{C}$. The inset shows a selected area diffraction pattern of the film taken along the [01-1] zone axis.

been presented by Gall et al. and Smith et al. to indicate that adatom mobility is much lower on the $\mathrm{ScN}$ (111) surface than on the $\operatorname{ScN}$ (100) surface. ${ }^{1,14}$ This effect is attributed to the higher density of dangling bonds on the (111), leading to a higher cation sticking coefficient. The intrinsically low adatom mobility (compounded by the use of low homologous temperatures during film deposition) is believed to result in the high roughness of (111)-oriented ScN films. A plot of roughness versus growth temperature for the films investigated in this study is shown in Fig. 4. A linear Arrhenius plot of the same data is also shown (Fig. 4, inset), indicating that the decrease in film roughness with increasing temperature is a result of a thermally activated process, which is consistent with the model of limited surface adatom mobility on the $\mathrm{ScN}$ (111) surface.

\section{B. Strain and the strain-free lattice parameter}

In order to determine actual strain values, knowledge of the strain-free lattice parameter is required. This should not be taken from existing literature, which gives a range of values from 4.500 to $4.505 \AA .^{2,15}$ The strain-free lattice pa-

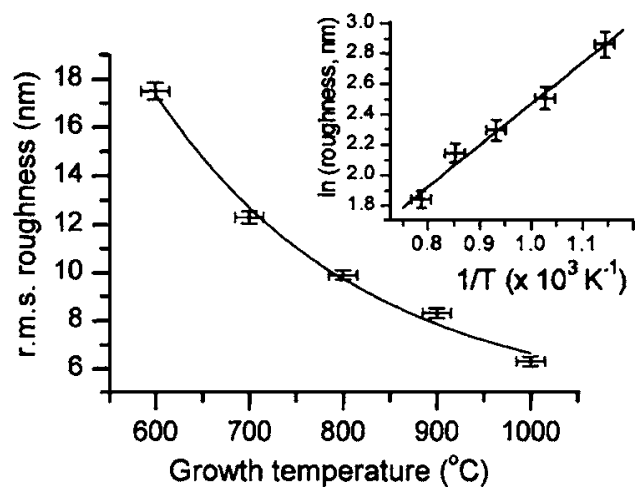

FIG. 4. Plot of rms surface roughness vs growth temperature of $225 \mathrm{~nm}$ thick ScN films. The line represents a first-order exponential fit. The inset shows a linear Arrhenius plot of the same data.

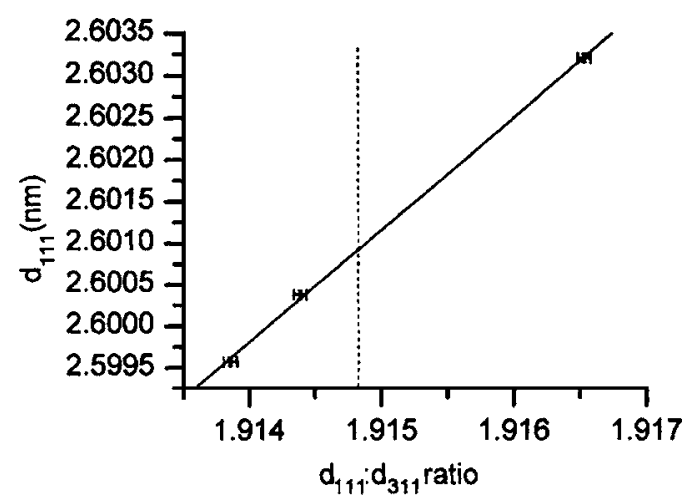

FIG. 5. Plot of $d_{111}: d_{311}$ ratio for films deposited under optimized conditions; the ideal ratio (corresponding to the strain-free unit cell) is denoted by dashed line.

rameter for our films was therefore found using a series of samples of different thicknesses grown under optimized conditions, which provided films of identical composition but under different levels of residual in-plane biaxial stress. The results are shown in Fig. 5, giving a value of 4.5047 $\pm 0.0005 \AA$ for $\mathrm{ScN}$ (according to the method outlined in Sec. II B). A similar plot for $d_{111}: d_{200}$ was made to confirm the results. This is within the range of strain-free lattice parameters [measured for both bulk material and (100)-oriented $\mathrm{ScN}$ films] mentioned previously. It is informative to consider the potential effect of stoichiometry on the film lattice parameter, given that the films studied in this investigation may have been deposited under slightly off-stoichiometric (possibly Sc-rich) conditions. It has already been determined that the most energetically favorable defect predicted to form under Sc-rich growth conditions is the $\mathrm{N}$ vacancy. However, even a $12 \% \mathrm{~N}$-vacancy concentration has been shown to give rise to just a $0.1 \%$ difference in lattice parameter compared to stoichiometric $\mathrm{ScN}{ }^{16}$ This level of difference is measurable using HRXRD. However, the electron concentration in different semiconducting $\mathrm{ScN}$ samples may very (due to both $\mathrm{N}$ vacancies and impurities), which is also known to affect the unit cell volume. ${ }^{17}$ This effect may partially account for the range of lattice parameters quoted previously. Even with highly accurate measurements, therefore, the $\mathrm{ScN}$ film stoichiometry cannot reliably be inferred from the lattice parameter.

In-plane and out-of-plane strains were then determined for the series of samples grown at different temperatures, and the series of samples of a range of thickness grown under optimized conditions. As shown in Fig. 6, in-plane strains were compressive for samples grown at $700{ }^{\circ} \mathrm{C}$ or below and tensile for those grown at $800{ }^{\circ} \mathrm{C}$ or above (average film thickness of $225 \mathrm{~nm}$ ). Some disagreement exists as to the value of the thermal expansion coefficient for $\mathrm{ScN}$ (4 $\times 10^{-6} \mathrm{~K}^{-1}$ is quoted by Gall et al. ${ }^{15}$ and $8 \times 10^{-6} \mathrm{~K}^{-1}$ is quoted by Dismukes et al. ${ }^{18}$ ) but it is consistently quoted at values higher than that of silicon $\left[2.6 \times 10^{-6} \mathrm{~K}^{-1}\right.$ (Ref. 19)]. Therefore, differential thermal contraction may be expected to lead to residual tensile strain in all films. Lattice-mismatch stress is not expected to play a large part as these relatively thick films are assumed to be relaxed. Point defects are also not expected to contribute to film stress, as the presence of $\mathrm{N}$ 


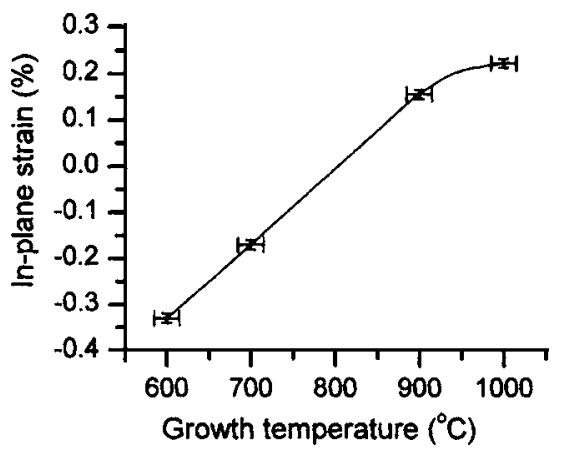

FIG. 6. Plot of in-plane strain vs growth temperature

vacancies (the only point defects expected in these films) has a negligible effect on the $\mathrm{ScN}$ lattice parameter, as previously discussed. The fact that films grown at lower temperatures are in compression indicates that an overall intrinsic compressive stress was developed during growth. The spread of literature values for the thermal expansion coefficient of $\mathrm{ScN}$ and the uncertainty regarding the degree of relief of the lattice mismatch strain at the interface prevents a quantitative determination of the magnitude of this stress, but it is clearly of the same order as that induced by differential thermal contraction for films of this thickness. Compressive stress can be induced during film growth by atomic peening, ${ }^{6}$ but when using $\mathrm{NH}_{3}$-MBE (molecular beam epitaxy) the filmforming species have only thermal energy, unlike radio frequency MBE or electron cyclotron resonance MBE which typically generate $\mathrm{N}_{2}{ }^{+}$species with high kinetic energies. The causes of compressive stress in this case may instead be related to a trend commonly observed in vapor-deposited thin films. Such films typically experience an initial intrinsic compressive stress, followed by the development of intrinsic tensile stress. A recovery leading to an overall compressive stress then occurs if the film grows to a sufficient thickness. ${ }^{20}$ This topic is discussed in greater detail in Ref. 20.

\section{Poisson's ratio}

Only data for those samples displaying relatively low strains have been considered, as these were assumed to be in the elastic (rather than the plastic) regime. The resulting plot comparing in-plane with out-of-plane strain is shown in Fig. 7, giving a value of Poisson's ratio of $0.188 \pm 0.002$ in the

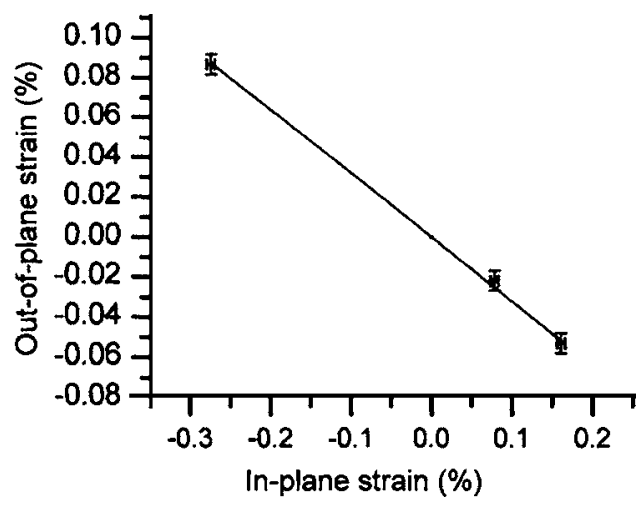

FIG. 7. Plot of in-plane vs out-of-plane strain; the slope of this plot is used to obtain Poisson's ratio. $\langle 111\rangle$ direction (using the method described in Sec. II B). This is marginally lower than the value of $0.20 \pm 0.01$ calculated for (100)-oriented single-crystal $\mathrm{ScN}$ films by Gall et al. ${ }^{15}$ however, due to the elastic anisotropy of transition metal nitrides, the values would be expected to differ.

\section{Stress determination and Young's modulus}

The Young's modulus for these films cannot be determined by nanoindentation because the film roughness is high with respect to the total film thickness, as indicated by TEM (Fig. 3). The indentation depth should extend substantially deeper than surface roughness in order to produce data representative of the bulk of the film, yet should not exceed $10 \%-20 \%$ of the film thickness to avoid the obtained values being affected by the elastic properties of the substrate. ${ }^{6}$ These conditions cannot be satisfied simultaneously for our films; therefore, the Young's modulus is determined using the procedure outlined in Sec. II C.

The average value of Young's modulus $270 \pm 25 \mathrm{GPa}$ which was obtained may be compared to the value of $302 \pm 15 \mathrm{GPa}$ reported from nanoindentation measurements by Gall et al., for single-crystal (100)-oriented films (grown under an incident $\mathrm{N}_{2}{ }^{+}$energy of $13 \mathrm{eV}$ ). ${ }^{14}$ Within the margin of error, these values are similar, despite the differences in growth method, film microstructure, and method of Young's modulus determination. This might be expected, as although the Young's modulus of the related material TiN is known to reach its maximum value in $\langle 100\rangle$ and its minimum value in $\langle 111\rangle,{ }^{21}$ the Young's moduli in those orientations are thought to differ only by a small amount. ${ }^{22}$ However, Gall et al. also reported a value of $356 \pm 20 \mathrm{GPa}$ for (100)-oriented $\mathrm{ScN}$ films grown using higher $\mathrm{N}_{2}{ }^{+}$energies of $20 \mathrm{eV}$. This is most likely due to an additional component of compressive stress induced during growth by atomic peening, ${ }^{6}$ which is expected to give rise to a higher value of Young's modulus. In contrast to the results of Gall et al., who reported compressive residual stresses from 2 to $12 \mathrm{GPa}$, levels of residual stress in our films are low. For films with similar thicknesses to those used in the study of Gall et al. ${ }^{14}$ (grown under optimized conditions at around $850{ }^{\circ} \mathrm{C}$ ) tensile residual stresses were not greater than $1 \mathrm{GPa}$. Films grown at lower temperatures displayed compressive residual stresses not greater than $1 \mathrm{GPa}$. The values were calculated from wafer curvature measurements using the value of Young's modulus obtained in this study. The attainment of low residual stress levels in $\mathrm{ScN}$ films on $\mathrm{Si}$ is promising for future device fabrication purposes.

\section{CONCLUSIONS}

HRXRD-based wafer curvature measurements and strain measurements have been combined to allow the sequential determination of the stress-free lattice parameter, the Poisson's ratio, and the Young's modulus of a series of (111)oriented ScN thin films of the same composition but of different thicknesses. Both the Poisson's ratio of $0.188 \pm 0.002$ and the Young's modulus of $270 \pm 25$ GPa were comparable to those previously determined for (100)-oriented $\mathrm{ScN}$, despite the elastic anisotropy of $\mathrm{ScN}$. Despite the difference in 
thermal expansion coefficients between $\mathrm{ScN}$ and $\mathrm{Si}$, the films are under low levels of residual stress, suggesting that subsequent growth of thin layers on top of the $\mathrm{ScN}$ films would be possible (a requirement for device fabrication). In order to decrease the surface roughness and avoid the development of a columnar film structure, higher adatom surface mobilities during growth are necessary. Further studies are currently under way to investigate the use of surfactants such as Ga, In, and As to improve the morphology of $\mathrm{ScN}$ films grown on silicon.

\section{ACKNOWLEDGMENTS}

The authors would like to thank Professor Bill Clyne for his helpful comments. This work was supported by the Engineering and Physical Sciences Research Council.

${ }^{1}$ A. R. Smith, H. A. H. Al-Brithen, D. C. Ingram, and D. Gall, J. Appl Phys. 90, 1809 (2001).

${ }^{2}$ H. Al-Brithen and A. R. Smith, Appl. Phys. Lett. 77, 2485 (2000).

${ }^{3}$ M. C. Johnson, E.-C. Bourret-Courchesne, J. Wu, Z. Lilienthal-Weber, D. N. Zakharov, R. J. Jorgenson, T. B. Ng, D. E. McCready, and J. R. Williams, J. Appl. Phys. 96, 1381 (2004).

${ }^{4}$ M. A. Moram, T. B. Joyce, P. R. Chalker, Z. H. Barber, and C. J. Humphreys, Appl. Surf. Sci. (in press).

${ }^{5}$ B. D. Cullity and S. R. Stock, Principles of X-Ray Diffraction, 3rd ed. (Prentice-Hall, Englewood Cliffs, NJ, 2003).
${ }^{6}$ M. Ohring, Materials Science of Thin Films, 2nd ed. (Academic, New York, 2002).

G. G. Stoney, Proc. R. Soc. London, Ser. A 82, 172 (1909).

${ }^{8}$ C. B. Masters and N. J. Salamon, Int. J. Eng. Sci. 31, 915 (1993).

${ }^{9}$ George E. Dieter, Mechanical Metallurgy, 3rd ed. (McGraw-Hill, New York, 1988).

${ }^{10}$ W. C. Oliver and G. M. Pharr, J. Mater. Res. 7, 1564 (1992).

${ }^{11}$ S. H. Jhi, J. Ihm, S. G. Louie, and M. L. Cohen, Nature (London) 399, 132 (1999).

${ }^{12}$ T. D. Moustakas, R. J. Molnar, and J. P. Dismukes, Proc.-Electrochem. Soc. 96-11, 197 (1996).

${ }^{13}$ D. Gall, I. Petrov, L. D. Madsen, J.-E. Sundgren, and J. E. Greene, J. Vac. Sci. Technol. A 16, 2411 (1998).

${ }^{14}$ D. Gall, I. Petrov, N. Hellgren, L. Hultman, J. E. Sundgren, and J. E. Greene, J. Appl. Phys. 84, 6034 (1998).

${ }^{15}$ D. Gall, I. Petrov, P. Desjardins, and J. E. Greene, J. Appl. Phys. 86, 5524 (1999).

${ }^{16}$ H. A. H. Al-Brithen, E. M. Trifan, D. C. Ingram, A. R. Smith, and D. Gall, J. Cryst. Growth 242, 345 (2002).

${ }^{17}$ M. Leszczynski, H. Teisseyre, T. Suski, I. Grzegory, M. Bockowski, J. Jun, S. Porowski, J. Bak-Misiuk, and J. Domagala, Nuovo Cimento Soc. Ital. Fis., D 19D, 585 (1997).

${ }^{18}$ J. P. Dismukes, W. M. Yim, and V. S. Ban, J. Cryst. Growth 13-14, 365 (1972).

${ }^{19}$ Yasumasa Okada and Y. Tokumaru, J. Appl. Phys. 56, 314 (1984).

${ }^{20}$ H. Windischmann, J. Vac. Sci. Technol. A 9, 2431 (1991).

${ }^{21}$ D. R. McKenzie, Y. Yin, W. D. McFall, and N. H. Hoang, J. Phys.: Condens. Matter 8, 5883 (1996).

${ }^{22}$ H. Ljungcrantz, M. Oden, L. Hultman, J. E. Greene, and J.-E. Sundgren, J. Appl. Phys. 80, 6725 (1996). 